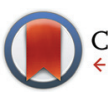

CrossMark \&lick for updates

Cite this: Polym. Chem., 2014, 5 , 6687

Received 8th July 2014

Accepted 6th August 2014

DOI: $10.1039 / c 4 p y 00941 j$

www.rsc.org/polymers

\title{
Surface modification of silicone via colloidal deposition of amphiphilic block copolymers $\uparrow$
}

\author{
Ghislaine Robert-Nicoud, ${ }^{a}$ Roberto Donno, ${ }^{a}$ Christopher J. Cadman, \\ Morgan R. Alexander ${ }^{c}$ and Nicola Tirelli ${ }^{a, d}$
}

\begin{abstract}
We report here on a method to functionalize silicone surfaces, which is based on the deposition of silicone-containing amphiphilic block copolymers from a colloidal water-ethanol dispersion. Using crosslinked silicones (Sylgard 184) as substrates, copolymers composed of two poly(glycerol monomethacrylate) (PGMMA) terminal blocks and a central poly(dimethylsiloxane) (PDMS) block can be effectively deposited when the PDMS content is $\geq 46 \mathrm{wt} \%$. ( $\geq 65 \mathrm{~mol} \%$ ); the deposition provides smooth and stable surfaces, which significantly affected the protein adsorption behaviour of the substrate, suggesting a possible application in biomaterial coating. In air, the block copolymer surface films underwent a reorganization, which differs from the classical hydrophobic recovery of silicones and may be related to a disordered folding of lamellar structures. This led to a predominant surface coverage by thin, possibly monomolecular layers, which displayed a non-restructuring polar surface. However, as a consequence of the reorganization also larger aggregates were produced, albeit in relatively small numbers; these aggregates underwent a progressive hydrophobization (in this case a hydrophobic recovery) and probably dominated the contact angle behaviour of the material. In summary, the colloidal deposition of amphiphilic silicone-based block copolymers successfully modifies the surface properties of silicone substrates; however, attention must paid to reorganization phenomena in order to maximize the stability of the coating.
\end{abstract}

\section{Introduction}

Silicone (poly(dimethylsiloxane), PDMS) artefacts are often employed for biomedical use in the form of medical devices ${ }^{1}$ such as intraocular lenses, ${ }^{2}$ urinary catheters ${ }^{3}$ and breast implants, ${ }^{4}$ but also microfluidic constructs; ${ }^{5}$ in their application they are often in contact with biological fluids, and the ensuing surface adsorption of proteins may lead to undesired outcomes, which in vivo can take the form of foreign body capsular reactions or of phenomena of platelet adhesion/activation. ${ }^{6,7}$ Another disadvantage of PDMS surfaces is the possibility of bacterial attachment, leading to the formation of biofilms, which are among the first promoters of chronic infections. ${ }^{3,8}$ Not surprisingly, much research aims to modify

${ }^{a}$ Institute of Inflammation and Repair/School of Medicine, University of Manchester, Oxford Road, Manchester, M13 9PT, UK. E-mail: nicola.tirelli@manchester.ac.uk; Fax: +44 (0)161 27523 96; Tel: +44 (0)161 2752480

${ }^{b}$ School of Pharmacy, University of Manchester, Oxford Road, Manchester, M13 9PT, UK

${ }^{c}$ School of Pharmacy, University of Nottingham, University Park, Nottingham, $N G 72 R D, U K$

${ }^{d}$ School of Materials, University of Manchester, Oxford Road, Manchester, M13 9PT, UK

$\dagger$ Electronic supplementary information (ESI) available. See DOI: 10.1039/ c4py00941j the surface behavior of silicones to improve their biomedical performance; for example, the fight against biofilms has led to the development of materials inherently resistant to bacterial attachment. ${ }^{9,10}$ Alternatively, the biomaterial can be modified only at its surface, without altering its bulk properties. For example, protein-repellent and biocompatible groups have been introduced on PDMS surfaces with a number of macromolecular structures, which include poly(ethylene glycol) (PEG) ${ }^{11-17}$ poly(2-methacryloyloxyethyl phosphorylcholine), ${ }^{18-20}$ poly(3-sulfopropyl methacrylate), ${ }^{21} \operatorname{poly}(N \text {-vinylpyrrolidone })^{22}$ and poly(2-hydroxyethyl methacrylate). ${ }^{23}$ Here, we have focused on a different hydrophilic structure that provides the advantageous combination of abundance of groups for chemical functionalization and the potential of a "stealth" behaviour: poly(2,3-dihydroxypropyl methacrylate), more commonly referred to as poly(glycerol monomethacrylate) (PGMMA), which features two vicinal alcohols on each repeating unit. While this diol group provides ease of functionalization, for example with pharmacologically active compounds, ${ }^{24}$ PGMMAbased hydrogels ${ }^{25-30}$ and surface layers ${ }^{28}$ have been shown to be non-toxic, protein-repellent, and non-cell-adhesive.

As for the functionalization, we have discarded the covalent attachment, either in a "grafted to" ${ }^{11,17}$ or in a "grafted from" (surface initiated polymerization) ${ }^{12,14,16,18,20-23}$ approach; as a common point, they would have required the introduction of 
reactive (polar) groups on the PDMS surface, whose reproducibility is typically marred by phenomena of hydrophobic recovery, ${ }^{31,32}$ with the migration of the polar groups into the PDMS bulk. Physical methods offer milder and less laborious conditions; some of them involve components that migrate to the surface or to the bulk depending on the exposure of the material to water or air, e.g. amphiphilic PDMS block copolymers, ${ }^{15,19}$ or Pluronic F127 dissolved in the PDMS bulk. ${ }^{33}$ Using a similar concept, i.e. the minimization of PDMS surface energy through a phase-segregated hydrophilic layer, amphiphilic polymers may be deposited only on the surface, thus avoiding the long-range morphological changes of a swelling-deswelling process. ${ }^{13,20}$ A well-known example of the latter approach is the adsorption of pluronic polymers from micellar dispersions, which has been applied to a variety of hydrophobic surfaces ${ }^{34,35}$ and, in particular, has been shown to increase the surface lubricity of silicone in a water environment. ${ }^{36}$

Here, we have followed the same general approach, employing ABA amphiphilic triblock copolymers; in order to increase the stability of the surface anchorage we have used PDMS as the central hydrophobic block, while using PGMMA for the terminal blocks. These polymers are hereafter referred to as Sil-GMMAs (Scheme 1) and were produced via ATRP of protected GMMA initiated by a bifunctional PDMS macroinitiator $^{37}$ to yield hydrophilic blocks of different lengths: their size, by influencing the exposure of the hydrophobic block and the curvature of the hydrophobe/water interface, should arguably be a major determinant of the physisorption efficiency.

\section{Experimental section}

\section{Materials}

A silicone elastomer kit (Sylgard 184, Dow Corning) was purchased from Elsworth Adhesives Ltd (Glasgow, UK). Bovine plasma fibrinogen Type I-S (BPF), bovine serum albumin (BSA), copper(I) chloride, dimethyl sulfoxide (DMSO), fluorescamine, hexamethyltriethylenetetramine (HMTETA), and sodium dodecyl sulphate (SDS) were purchased from SigmaAldrich (Gillingham, UK). Phosphate buffer saline (PBS) was bought from Oxoid (Basingstoke, UK). Acetic acid, dioxane, ethanol, hexane, methanol and tetrahydrofuran were purchased from Fisher Scientific (Loughborough, UK). Deionized water, ethanol and hexane were passed through a $0.22 \mu \mathrm{m}$ filter before use. Bis-trimethylsilyl GMMA and 2-bromoisobutyrylethoxypropyl terminated PDMS were prepared according to a previously reported procedure. ${ }^{37}$

\section{Physico-chemical characterization}

IR spectroscopy. Fourier transform infrared (FT-IR) spectra were recorded in attenuated total reflectance mode on a Tensor 27 Bruker spectrometer.

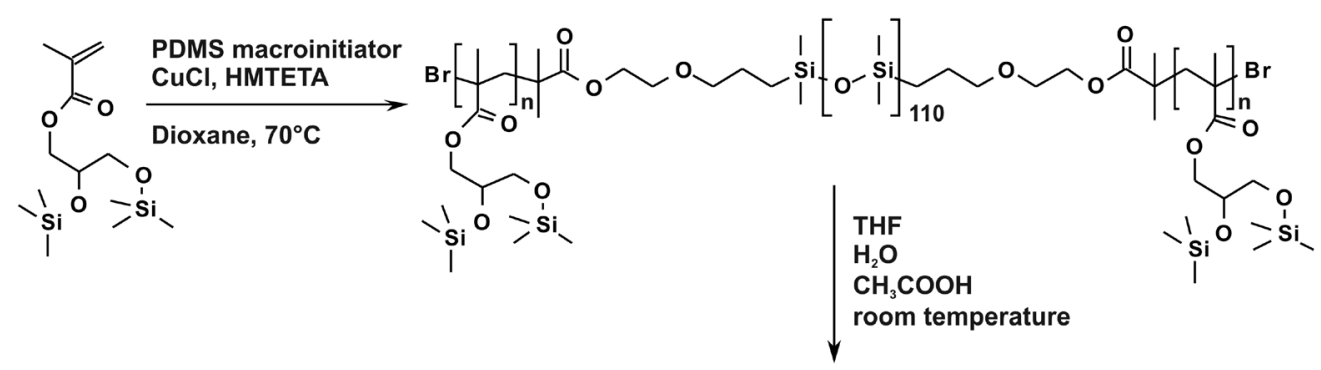

\begin{tabular}{ccc}
\hline \multirow{2}{*}{ Polymer } & \multicolumn{2}{c}{ Molar composition } \\
& GMMA & DMS \\
\hline SilGMMA1 & 26 & 74 \\
SilGMMA2 & 34 & 66 \\
SilGMMA3 & 42 & 58 \\
SilGMMA4 & 50 & 50 \\
SilGMMA5 & 58 & 42 \\
\hline
\end{tabular}

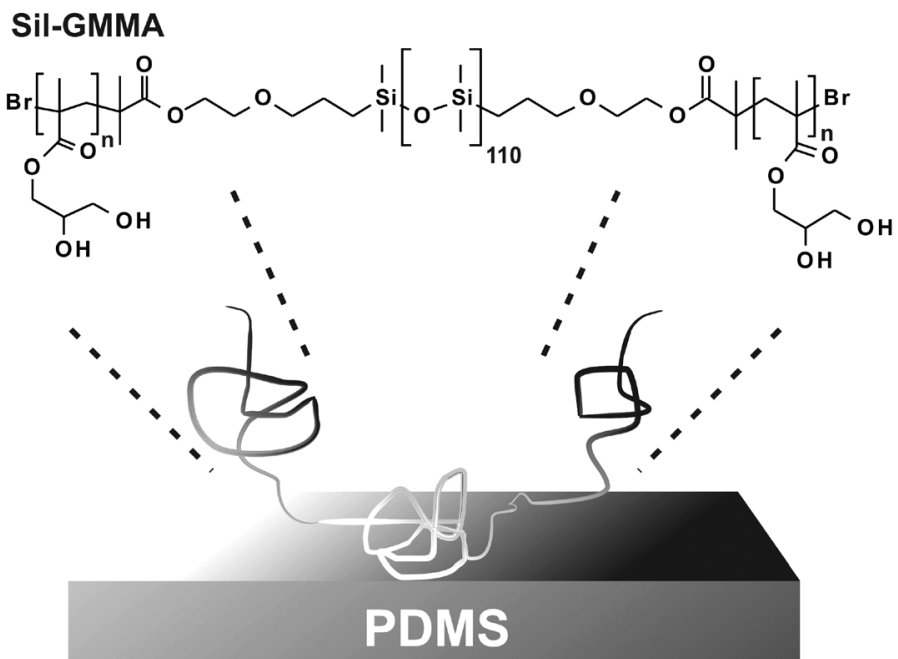

Scheme 1 Sil-GMMA triblock copolymers were synthesized in a protected form via ATRP using a PDMS macroinitiator and silylated GMMA as a monomer. The hydrolysis of the trimethylsilyl groups provided the final amphiphilic macromolecules. The table provides the molar ratio between GMMA and DMS units in the polymer, calculated from the ratio of the ${ }^{1} \mathrm{H}$ NMR resonance of the PGMMA chain group $\left(\mathrm{CH}_{3}\right.$ at $\left.0.7-1.2 \mathrm{ppm}\right)$ and the resonance of the PDMS chain $\left(\mathrm{CH}_{3}\right.$ at $\left.-0.06 \mathrm{ppm}\right)$. 
Dynamic light scattering (DLS). Size measurements were performed using a Zetasizer Nano ZS instrument (Model ZEN2500, Malvern Instruments Ltd, UK) at a scattering angle of $173^{\circ}$ and at a temperature of $25{ }^{\circ} \mathrm{C}$. The values of hydrodynamic diameter (which corresponds to the weighted $Z$-average) and polydispersity (PD) were obtained from a cumulant analysis algorithm wherein a single particle size is assumed and a single exponential fit is applied to the autocorrelation (Zetasizer Nano Software, Malvern Instruments Ltd, UK). The colloidal dispersions were prepared at a concentration of $10 \mathrm{mg} \mathrm{mL}^{-1}$ in filtered water and ethanol and were not filtered after preparation.

Mechanical characterization. Silicone substrates were analyzed using a texture analyzer (Model TA-XT plus, Stable Micro System Ltd, UK) in compression mode applying a maximum stress of $1.77 \mathrm{MPa}\left(F_{\max }=50 \mathrm{~N}\right)$. The elastic modulus was obtained from the slope of the plot of stress $(F / A)$ versus strain $(\Delta L / L)$ where $F$ is force, $A$ is the contact area with the probe $\left(0.28 \mathrm{~cm}^{2}\right), L$ is the height of the sample and $\Delta L$ is the change in height. Stress-strain curves were fitted in their linear region, where typical strains were in the range of $0.04-0.4$ for stiff gels and 0.02-0.2 for soft gels. The data reported here are the mean and standard deviation of at least 3 different substrates.

Water contact angle. The sessile drop method was used to measure water contact angles with a Krüss 100-DSA (drop shape analysis). All samples were dried in air for $30 \mathrm{~min}$ before measurements. The advancing contact angle was measured immediately after deposition of the water drop on the surface while the receding contact angle was measured after the water drop was left to evaporate for 3 minutes. The values reported here are the results of at least 3 drops on each surface.

X-Ray photoelectron spectroscopy (XPS). Spectra were collected on a Kratos ULTRA-DLD instrument configured with a monochromated $\mathrm{Al} \mathrm{K} \mathrm{K}_{\alpha}(1486.6 \mathrm{eV})$ X-ray source. Measurements were carried out on electron taking-off angles (t.o.a.) from the sample surface of $90^{\circ}$ and $10^{\circ}$. Survey spectra and high sensitivity scans of the $\mathrm{Br} 3 \mathrm{~d}$ area were obtained at a pass energy of $80 \mathrm{eV}$. The pass energy was $20 \mathrm{eV}$ for the narrow scans. All data analyses were accomplished using CasaXPS software. The curve fittings of the high resolution peaks were carried out using a combination of Gaussian and Lorentzian functions (70:30\%). The binding energies were charge corrected to the $\mathrm{C} 1 \mathrm{~s}$ binding energy of PDMS $\mathrm{CH}_{3}$ groups, using $284.5 \mathrm{eV}$ as the energy associated with double methyl-substituted silicon atoms. ${ }^{38}$

Atomic force microscopy (AFM). All measurements were performed at $25{ }^{\circ} \mathrm{C}$ using a Molecular Force Probe 3D AFM (MFP-3D, Asylum Research, Santa Barbara, CA). The nominal parameters of the silicon cantilevers (model AC160TS, Olympus) used in this study were: spring constant $=42 \mathrm{~N} \mathrm{~m}^{-1}$, tip radius $<10 \mathrm{~nm}$, tip angle $=35^{\circ}$, tip height $=7-15 \mu \mathrm{m}$, resonance frequency $=300 \mathrm{kHz}$.

(A) Imaging. Tapping mode parameters: scan frequency $=$ $1 \mathrm{~Hz}\left(25.70 \mu \mathrm{m} \mathrm{s}^{-1}\right)$, gain $=10$, setpoint values $550 \mathrm{mV}-$ $650 \mathrm{mV}$, resonance frequency $313-344 \mathrm{kHz}$ in air and 150-175 kHz in deionized water. Three different $10 \mu \mathrm{m} \times$
$10 \mu \mathrm{m}$ regions of each sample (unmodified and coated S10, S20) were imaged. The original images (without any operations of flattening or filtering) were analyzed with Igor-pro (Asylum Research AFM software, Version 101010 + 1202, Wavemetrics, Portland, OR) in order to calculate the root mean square (RMS) roughness. All height images displaying both flat areas and aggregates were segmented to analyze separately the two regions. The segmentation was obtained by applying a threshold of $10 \mathrm{~nm}$ on the different height images (see ESI, Fig. 5SI $\dagger$ ).

(B) Nanoindentation. Measurements on mica surfaces were used to calibrate the deflection sensitivity of the cantilever, which is necessary to convert force-displacement measurements into force-indentation dependences. The spring constant of the cantilevers was calculated with the thermal method $^{39}$ and ranged between $36.2 \mathrm{~N} \mathrm{~m}^{-1}$ and $43.7 \mathrm{~N} \mathrm{~m}^{-1}$. The deflection sensitivity ranged between $65 \mathrm{~nm} \mathrm{~V}^{-1}$ and $75 \mathrm{~nm} \mathrm{~V}^{-1}$. Three different $10 \mu \mathrm{m} \times 10 \mu \mathrm{m}$ regions were investigated for each sample obtaining force maps for each region with a spatial resolution of $0.25 \mu \mathrm{m}^{2}(20 \times 20$ curves on an area of $100 \mu \mathrm{m}^{2}$ ). All force curves were acquired with a loading/ unloading rate equal to $6 \mu \mathrm{m} \mathrm{s}^{-1}$ (frequency $=1 \mathrm{~Hz}$ ) and the maximum force applied on the surface of each samples was $500 \mathrm{nN}$.

Assuming a Hookean behaviour for the silicon cantilever, force-displacement data were converted to force-indentation plots. The same software was used to fit all the force curves in order to calculate Young's modulus; the power law exponent $(\beta)$ of the data ranged between 1.80 and 2.00, suggesting the use of a Hertz-cone model (punch model: $\beta=1$, Hertz-sphere model: $\beta=1.5$, Hertz-cone model: $\beta=2 ; 0.5 \geq \beta \geq 2.5$ means that the Hertz model cannot be used to fit the data due to excessive adhesion forces), which is described by the following equation: ${ }^{40,41}$

$$
F=\frac{2}{\pi} E^{*} h^{2} \tan \alpha
$$

where $F$ is the load, $h$ is the displacement of the specimen surface (indentation), $\alpha$ is the half opening apex-angle of the tip and $E^{*}$ is the combined modulus, defined as follows: ${ }^{42}$

$$
\frac{1}{E^{*}}=\frac{\left(1-\nu_{\mathrm{s}}^{2}\right)}{E_{\mathrm{s}}}+\frac{\left(1-\nu_{\mathrm{t}}^{2}\right)}{E_{\mathrm{t}}}
$$

where $E_{\mathrm{s}}, \nu_{\mathrm{s}}$ and $E_{\mathrm{t}}, \nu_{\mathrm{t}}$ are Young's moduli and Poisson's ratios for the sample and the tip, respectively. $E_{\mathrm{t}}=150 \mathrm{GPa}, \nu_{\mathrm{t}}=0.17$, $\alpha=18^{\circ}$ and $\nu_{\mathrm{s}}=0.5 .^{43}$

The adhesion forces between the AFM tip and the sample surface were determined both in loading (indentation) and unloading (retraction) force curves. The contact point was identified as the minimum of the loading curve. Upon retraction, the tip maintains the contact with the surface until the cantilever force overcomes the adhesion force: at this point the force reaches a minimum and then rapidly decreases to 0 (the tip is pulled out of contact with the surface). The difference between the minimum (negative) force and the force felt at a large distance from the surface is the adhesion force. 
Protein adsorption tests. PDMS substrates were cured as described in the Preparative Procedures in a tissue-culture polystyrene (TCPS) 24-well plate or in a black TCPS 96-well plate (BD Falcon) to perform, respectively, bicinchoninic acid (BCA) or fluorescamine assays. $1 \mathrm{~mL}$ (BCA) or $0.1 \mathrm{~mL}$ (fluorescamine) of protein solution in PBS at pH $7.3\left(1 \mathrm{mg} \mathrm{mL} \mathrm{m}^{-1} \mathrm{BPF} /\right.$ 1 and $10 \mathrm{mg} \mathrm{mL}^{-1}$ BSA for BCA; $0.1,0.5$ and $1 \mathrm{mg} \mathrm{mL}^{-1} \mathrm{BPF} /$ $0.1,1$ and $10 \mathrm{mg} \mathrm{mL}^{-1} \mathrm{BSA}$ for fluorescamine) was poured into each well and incubated at $37^{\circ} \mathrm{C}$ for $3 \mathrm{~h}$. The protein solution was then removed and the wells were washed with fresh PBS twice. BCA: after adding $0.55 \mathrm{~mL}$ of SDS water solution $(10 \mathrm{mg}$ $\mathrm{mL}^{-1}$ ), the plate was sonicated for $20 \mathrm{~min}$ at room temperature. The protein concentration in the SDS solution was then determined using a QuantiPro ${ }^{\mathrm{TM}}$ BCA protein assay kit, reading the absorbance of the BCA-Cu${ }^{\mathrm{I}}$ complex at $562 \mathrm{~nm}$ with a BioTek Synergy 2 multi-mode microplate reader (3 samples, 4 measurements per sample). Fluorescamine: $0.075 \mathrm{~mL}$ of $2 \mathrm{mg} \mathrm{mL} \mathrm{m}^{-1}$ fluorescamine solution in DMSO and $0.075 \mathrm{~mL}$ of PBS ( $\mathrm{pH}$ 7.3) were poured into each well. The fluorescence was read on a Bio-Tek Synergy 2 multi-mode microplate reader using filters for excitation at $360 \pm 40 \mathrm{~nm}$ and for emission at $460 \pm 40 \mathrm{~nm}$ after $15 \mathrm{~min}$ (mean and standard deviation of the results from 12 samples).

\section{Preparative procedures}

Substrate preparation. The elastomer kit Sylgard 184 consists of a "base" and a "cross-linker", which are respectively a silane (Si-H)-containing poly(dimethyl siloxane) and tetramethyl tetravinyl cyclotetrasiloxane. PDMS substrates with different cross-linking densities were prepared by mixing the base and the cross-linker at various weight ratios $(5: 1,10: 1$, $20: 1$ and 50:1 corresponding to the substrates S5, S10, S20 and S50 respectively), degassed under reduced pressure for $30 \mathrm{~min}$ and transferred into TCPS Petri dishes. The PDMS films were cured at room temperature for 2 days and disks with a diameter of $6 \mathrm{~mm}$ (samples for the texture analyzer) or $9 \mathrm{~mm}$ (substrates for adsorption experiments) and a thickness of approximately $3 \mathrm{~mm}$ were cut using cork borers. The substrates were washed with hexane to remove any unreacted oligomers and dried under reduced pressure.

Synthesis of Sil-GMMA copolymers. Sil-GMMA polymers were prepared according to a previously reported procedure. ${ }^{37}$ In a typical synthesis (Sil-GMMA1) a mixture of $500 \mathrm{mg}$ of 2-bromoisobutyrylethoxypropyl terminated PDMS (PDMS macroinitiator, $8000 \mathrm{~g} \mathrm{~mol}^{-1}$ ), bis-trimethylsilyl GMMA (751 mg, $2.47 \mathrm{mmol}$, corresponding to 20 equivalents to bromoisobutyryl groups) and HMTETA (29 mg, $0.12 \mathrm{mmol}$, corresponding to 1 equivalent to bromoisobutyryl groups) were degassed by bubbling argon for 45 minutes followed by the addition of degassed dioxane $(1.3 \mathrm{~mL})$. Copper(I) chloride (12 mg, $0.12 \mathrm{mmol}$, corresponding to 1 equivalent to bromoisobutyryl groups) was then added under an inert atmosphere. The solution was stirred at $70{ }^{\circ} \mathrm{C}$ for $4 \mathrm{~h}$. The polymer was purified by three precipitations in ice cold methanol. The bistrimethylsilylated group was then hydrolyzed by dissolving $100 \mathrm{mg}$ of the polymer in $1 \mathrm{~mL}$ of tetrahydrofuran and adding
$0.1 \mathrm{~mL}$ of water and $0.5 \mathrm{~mL}$ of acetic acid. After $4 \mathrm{~h}$, the precipitated polymer was collected, redissolved in DMSO and purified by dialysis (3500 $\mathrm{g} \mathrm{mol}^{-1}$ molecular weight cutoff) in water. After freeze-drying, a white block copolymer was obtained. Different compositions of block copolymers were synthesized using the same PDMS macroinitiator and varying the feed ratio of bis-trimethylsilyl GMMA monomers.

Adsorption of block copolymers. Block copolymers with various block ratios were dissolved in ethanol at different concentrations (40, 20 and $10 \mathrm{mg} \mathrm{mL}{ }^{-1}$ ) and then diluted to respectively 20, 10 and $5 \mathrm{mg} \mathrm{mL}^{-1}$ with water. The PDMS elastomer disks were then exposed to $1 \mathrm{~mL}$ of each polymer solution for 3 days and washed with fresh water. In order to assess the long term stability of the surface modifications, disks were then stored in air or water for 14 days.

\section{Results and discussion}

\section{Characterization of the uncoated substrates}

We have produced silicone substrates using the popular twocomponent formulation Sylgard 184; this is composed of a base (mostly vinylated PDMS and vinylated or trimethylsilylated silica) and a cross-linker (silane-containing PDMS + vinylated PDMS and vinylated or trimethylsilylated silica + tetramethyl tetravinyl cyclotetrasiloxane), ${ }^{44}$ which we have used at weight ratios between 10:1 (S10) and 50:1 (S50). Please note that cross-link density influence both mechanical and surface properties (Table 1).

Morphology. S10 and S20 presented extremely smooth surfaces, with RMS roughness below $1 \mathrm{~nm}$ (Table 1 and Fig. 1, top); the analysis of samples produced with lower amounts of cross-linker provided unreliable results (S50), possibly due to the incomplete cross-linking leading to adhesion of the material to the AFM tip and its contamination.

Mechanical properties. Both in compression and nanoindentation the elastic modulus scaled approximately linearly with the amount of cross-linker, varying from 0.095 (S50 - only compression) to $2.90 / 2.1 \mathrm{MPa}$ (S10, resp. in compression and nanoindentation). It also showed a remarkable spatial homogeneity, with variations $<5 \%$ in $10 \mu \mathrm{m} \times 10 \mu \mathrm{m}$ areas (Fig. 1, middle). Higher cross-link densities did not provide appreciably higher moduli: for example a $5: 1$ ratio gave $E=2.74 \pm$ $0.10 \mathrm{MPa}$; lower cross-link densities, i.e. $>50: 1$ ratios, provided materials with a predominantly viscous behaviour. Exposure to water did not significantly affect the modulus, as it would be expected for a strongly hydrophobic and thus non-swellable material (Table 1, data in air (a) and in water (w)).

Surface properties. The cross-linker has a lower number (either per volume or per weight) of methyl groups in comparison with the macromonomer; therefore, due to the low surface energy of the $\mathrm{CH}_{3}$ group, less cross-linked materials are likely to be more hydrophobic. Indeed the water contact angle increased with decreasing cross-linker content. Despite some significant heterogeneities (Fig. 1, bottom) the adhesion force of the polar AFM tip to the silicone surface also increased with 
Table 1 Mechanical and surface properties determined by compression tests, AFM/nanoindentation and water contact angle for PDMS elastomers with different cross-link densities

\begin{tabular}{|c|c|c|c|c|c|c|c|}
\hline \multirow[b]{2}{*}{ Sample $^{a}$} & \multicolumn{2}{|c|}{ Young's modulus (MPa) } & \multicolumn{2}{|c|}{ Contact angle $\left(^{\circ}\right)$} & \multicolumn{2}{|c|}{ Adhesion force $^{d}(\mathrm{nN})$} & \multirow[b]{2}{*}{$\begin{array}{l}\text { Roughness } \\
(\mathrm{nm})\end{array}$} \\
\hline & Compression $^{b}$ & $\begin{array}{l}\text { Nanoindentation }{ }^{c} \\
\text { (air/water) }\end{array}$ & Advancing & Receding & $\begin{array}{l}\text { Loading } \\
\text { (air/water) }\end{array}$ & Unloading & \\
\hline $\mathrm{S} 10(10: 1)$ & $2.90 \pm 0.05$ & $\begin{array}{l}2.11 \pm 0.02(\mathrm{a}) \\
2.05 \pm 0.03(\mathrm{w})\end{array}$ & $105 \pm 1$ & $103 \pm 1$ & $<0.1^{e} e^{7.6 \pm 0.6}$ & $\begin{aligned} 14.7 & \pm 1.2(\mathrm{a}) \\
102 & \pm 5(\mathrm{w})\end{aligned}$ & $0.54 \pm 0.10$ \\
\hline S20 (20:1) & $0.76 \pm 0.05$ & $\begin{array}{l}1.14 \pm 0.04(\mathrm{a}) \\
1.08 \pm 0.04(\mathrm{w})\end{array}$ & $112 \pm 2$ & $108 \pm 4$ & $\begin{array}{l}11.2 \pm 0.8 \\
<0.1^{e}\end{array}$ & $\begin{aligned} 34.7 & \pm 2.3(\mathrm{a}) \\
115 & \pm 7(\mathrm{w})\end{aligned}$ & $0.49 \pm 0.11$ \\
\hline
\end{tabular}

${ }^{a}$ In brackets the base : X-linker weight ratio. ${ }^{b}$ Measured in air on cylindrical samples on an area of approx. $28 \mathrm{~mm}^{2} .{ }^{c}$ Measured both in air and in water with AFM in nanoindentation mode. ${ }^{d}$ Measured both in air and in water as the maximum negative force recorded in the indentation (loading) and in the retraction (unloading) curves. No loading adhesion forces were observed in water. The adhesion force in loading is often referred to as the van der Waals component of the total adhesion. See also ESI, Fig. 1SI. ${ }^{e}$ The negligible adhesion in loading is due to the presence of a film of water that shields the tip from the substrate surface. ${ }^{f}$ S50 could not be analyzed via nanoindentation (tip rapidly covered by debris).

decreasing cross-linker content. This is likely an artifact due to the larger deformability of S20, which increases the contact area between the tip and the substrate during indentation; therefore the higher adhesion force would not be an indication of a higher polarity of the surface (in accordance with contact angle data).

Surface reorganization. in water, the adhesion force between the polar AFM tip and silicone substrate was 4-5 times higher than in air, which is an indication that the PDMS surface is more hydrophilic surface under water, i.e. a hydrophobic recovery is likely to occur in air. Contact angle data do not confirm this point, likely due to the experimental conditions used: advancing and receding contact angles are only marginally different, but receding angles are measured only after a few seconds of exposure to water and this may not allow a sufficient time for the polar groups to re-surface.

\section{Surface modification via colloidal deposition}

We have employed ethanol-water dispersions: ethanol has already been used to deposit block copolymers on silicone ${ }^{20}$ and we hypothesized that its combination with water would allow at the same time for a thermodynamic driving force (the high surface energy of silicone in water being reduced by coating) and for kinetically favourable conditions (increased solubility of polymers in the medium leading to accelerated deposition). It is worth mentioning that deposition from pure water did not appear to have much success.

Always with the aim to accelerate the deposition, we have considered the time dependence of the size of Sil-GMMA aggregates as an indication of their dynamics; using 9:1 and 1:1 water-ethanol (see ESI, Fig. 2SI $\dagger$ ), the aggregate size increased with increasing PDMS content, as it happens also in water, while in the first mixture the aggregates were rather stable for up to 4 weeks, in 1:1 ethanol-water they showed quite a marked evolution (decrease with time), which led us to select the latter solvent composition as the most "dynamic" and to use it for all deposition experiments.

Infrared spectroscopy. The deposited amount of SilGMMA1-5 was evaluated using ATR-IR spectroscopy (Fig. 2); the intensity of the $\mathrm{C}=\mathrm{O}$ stretching peak was used as a semi- quantitative indication of the amount of GMMA-containing material deposited on the surface. For the polymers with the highest PGMMA content, the peak could be barely seen and therefore Sil-GMMA4 and 5 were not further used.

Water contact angle. Advancing and receding water contact angles were recorded on freshly modified substrates and after two weeks of storage in air or in water (Fig. 3). The deposition experiments were performed at a concentration of $10 \mathrm{mg}$ $\mathrm{mL}^{-1}$, but it is noteworthy that, in the range $5-20 \mathrm{mg} \mathrm{mL}^{-1}$, the polymer concentration did not affect the contact angle results (see ESI, Fig. $3 \mathrm{SI} \dagger$ ), suggesting a rather complete coverage (saturation) of the silicone surface.

Sil-GMMA3 (Fig. 3A and B) and S50 (Fig. 3C and D) were discarded, respectively, as a coating agent and as a substrate, because the corresponding coating processes did not lead to any appreciably difference of the contact angles, suggesting negligible and/or unstable deposition: this is hardly surprising for Sil-GMMA3, which forms more stable and higher curvature aggregates in water and therefore is possibly more prone to desorption. In contrast, the insignificant effects on S50 may be related to the ease of migration of the polymers from the surface to the bulk, due to the low cross-link density of the material. Please note that further experiments were therefore conducted with Sil-GMMA1 and 2, i.e. employing S10 and S20 as the substrates; the identity of the polymer (Fig. 3A and B) and that of the substrate (Fig. 3C and D) did not appear to have a major effect. It is also noticeable that (A) the adsorption of Sil-GMMA1 and 2 lowered more the receding than the advancing contact angles, (B) lower contact angles were recorded upon storage in water or immediately after preparation whereas both advancing and receding angles were indistinguishable from those of the unmodified substrates upon prolonged storage in air (Fig. 3A and B). These two phenomena would hint at re-organization phenomena occurring on these coated surfaces when in air; it is also noteworthy that the lowest contact angles showed large errors, suggesting the presence of heterogeneities.

XPS. The deposition of Sil-GMMA1 and 2 on S10 was further analyzed using XPS (Table 2). The composition of the 


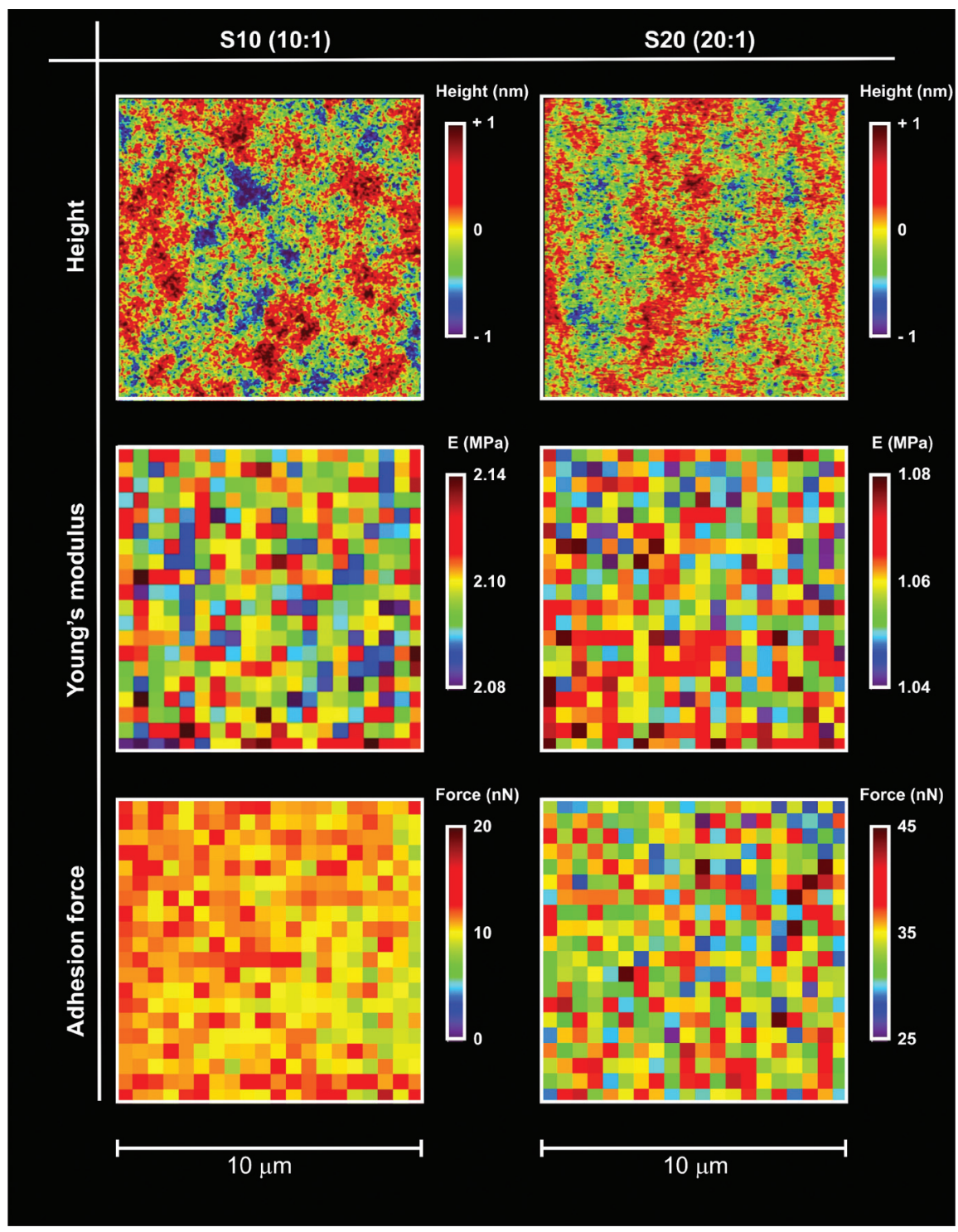

Fig. 1 Typical height, modulus and adhesion maps for S10 and S20 samples in air; the maps were obtained using AFM in tapping mode (top), or nanoindentation (middle and bottom). The nanoindentation maps report the Young's modulus (middle) calculated using a Hertzian model and the adhesion force (bottom) calculated from retraction curves (see for example ESI, Fig. 1SI $\dagger$ right). In both cases, the maps showed the PDMS surfaces to be very homogeneous at least at the resolution used $(500 \mathrm{~nm}: 20$ points on a $10 \mu \mathrm{m}$ scale). It is noted that, although characterized by similarly smooth surfaces, the two materials drastically differed in modulus and polarity (lower modulus and higher adhesion recorded for S20).

upper layers of unmodified S10 (take-off angle (t.o.a.) $=10^{\circ}$ ) was considerably different from what was expected from pure PDMS, with a higher oxygen content independently of the storage conditions (air or water); the composition of deeper layers (t.o.a. $=90^{\circ}$ ) was, in contrast, closer to that of PDMS. The group of Vancso obtained similar results on Sylgard 184 with the same base/cross-linker ratio ${ }^{45,46}$ and interpreted them as due to silica fillers partially segregated during cross-linking.

Due to the large ( $\geq 66$ wt $\%$ ) PDMS content of the block copolymers the surface composition of the coated samples is expected to undergo only moderate changes, with some increase in the carbon content and decrease in the silicon one; indeed only rather small differences were recorded (Table 2), with the silicon content appearing slightly reduced at t.o.a. = $10^{\circ}$. In a further analysis, high resolution C 1s spectra clearly showed the presence of carbonyl groups (peak at $289.1 \mathrm{eV}$, shift of $3.8 \mathrm{eV}$ relative to saturated hydrocarbon) ${ }^{48}$ (Fig. 4B, compared to Fig. 4A), which was accompanied by an increase in the $\mathrm{C}-\mathrm{O}$ signal.

$\mathrm{Br}$ atoms were recorded for Sil-GMMA1 at a t.o.a. of $10^{\circ}$ (see ESI, Fig. 4SI $\dagger$ ), while they were absent at t.o.a. $=90^{\circ}$ on coated samples. XPS therefore confirmed the presence of small amounts of Sil-GMMA1 and 2 and only in the upper surface layers. Storage in water affected in an equal fashion both uncoated and coated substrates, indicating that their upper layers may be slightly less rich in silicone, which would possibly support some hydrophobic recovery; however, the effect on both uncoated and coated samples was rather marginal. 


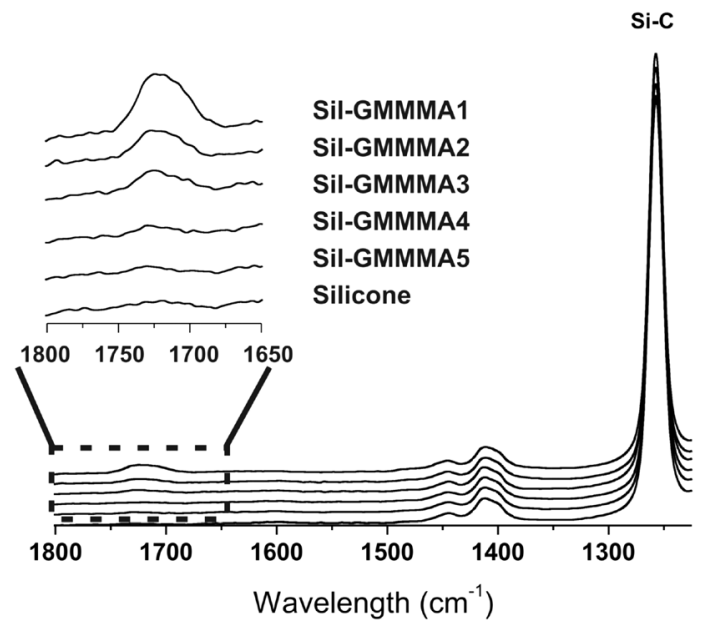

Fig. 2 ATR-IR spectra of S10 unmodified and after 3 day exposure to water dispersions of Sil-GMMA1 to $5\left(10 \mathrm{mg} \mathrm{mL}^{-1}\right.$ in EtOH- $\left.\mathrm{H}_{2} \mathrm{O} 1: 1\right)$. The insert shows the $\mathrm{C}=\mathrm{O}$ stretching region. The low intensity of the carbonyl absorption, e.g. in comparison with that of $\mathrm{Si}-\mathrm{C}$ stretching from the underlying silicone, is due to the thinness of the polymer layers.
AFM. Under water (Fig. 5B), Sil-GMMA1-coated S10 exhibited a smooth profile (roughness 1-2 nm) and a very homogeneous phase. Upon drying (Fig. 6C), the majority of the surface retained a roughness similar to that shown in water $(<5 \mathrm{~nm})$, but exhibited a characteristic pattern both in phase and height (Fig. 6); the apparently more rigid (larger phase) component seemed to produce $\sim 2 \mathrm{~nm}$ thick domains on the top of the lower phase component (in black in the phase picture of Fig. 6). Due to both its deeper localization and the likely softer character (lower phase value) it seems reasonable to identify the lower phase as PDMS, and thus the top component as PGMMA. In addition to these flat, patterned areas, some micron-sized structures appeared upon drying; they showed an irregular profile and a roughness in hundreds of nanometers. Both the irregular structures and the flat areas disappeared upon a second exposure to water (Fig. 5D) and partially re-appeared after a second drying stage (Fig. 5E). Due to their larger size and their absence under water, the large aggregates cannot be identified as the colloids used in the deposition and must be produced during drying.

We examined in more detail the kinetics of the drying process.

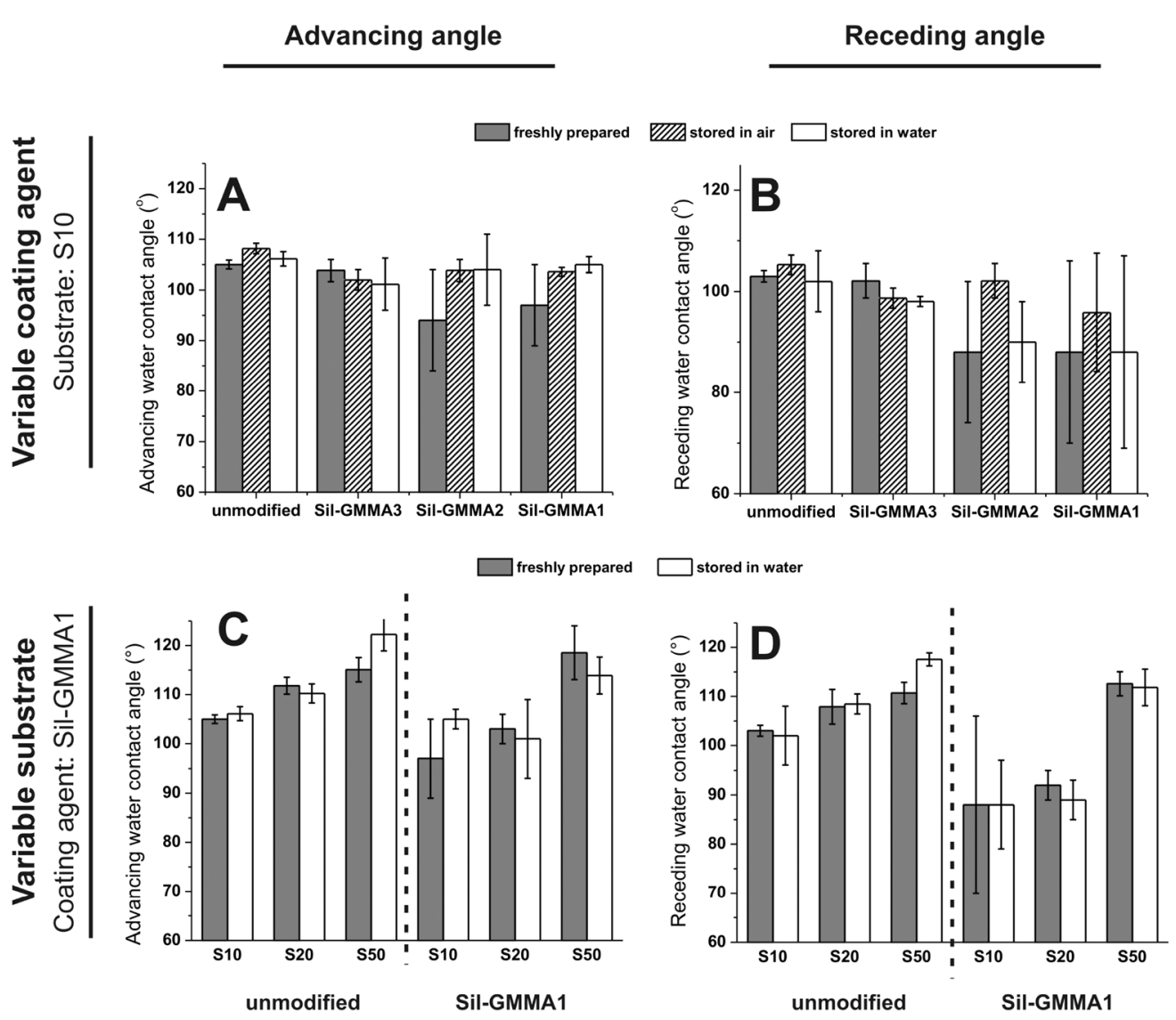

Fig. 3 Top: advancing (A) and receding (B) contact angles of S10, unmodified and after a 3-day exposure to $10 \mathrm{mg} \mathrm{mL}^{-1}$ Sil-GMMA1-3 dispersion in $1: 1$ ethanol-water. The measurements were performed on freshly prepared samples (grey columns) and after storage in air (patterned columns) or water for 14 days (white columns). Bottom: advancing (C) and receding (D) water contact angles of substrates with different cross-link densities, unmodified and after a 3-day exposure to $10 \mathrm{mg} \mathrm{mL}^{-1}$ Sil-GMMA1 dispersion in 1:1 ethanol-water. The measurements were performed on freshly prepared samples (grey columns) and after storage in water for 14 days (white columns). 
Table 2 Surface composition (\% atom) of silicone disks (S10) unmodified and treated with Sil-GMMA1-2 copolymers after storage for 14 days in air or water

\begin{tabular}{|c|c|c|c|c|c|c|c|c|}
\hline \multicolumn{2}{|l|}{ Sample } & \multicolumn{2}{|l|}{$\mathrm{C}^{a}$} & \multicolumn{2}{|l|}{$\mathrm{O}^{a}$} & \multicolumn{2}{|l|}{$\mathrm{Si}^{a}$} & $\mathrm{Br}^{a}$ \\
\hline Sil-GMMA1 & & 57 & & 31 & & 12 & & 0.2 \\
\hline S10 (unmodified) & Stored in air & 47 & 47 & 30 & 26 & 23 & 26 & 0 \\
\hline Sil-GMMA1 & & 48 & 49 & 30 & 25 & 21 & 26 & 0.02 \\
\hline Sil-GMMA2 & & 49 & 50 & 30 & 24 & 21 & 27 & 0 \\
\hline S10 (unmodified) & Stored in water & 49 & 49 & 30 & 24 & 21 & 27 & 0 \\
\hline
\end{tabular}

${ }^{a}$ t.o.a. $=10^{\circ}$ (left) and $90^{\circ}$ (right). Considering that $95 \%$ of the observed photoelectron signal derives from a layer thickness $3 \lambda$ sin(t.o.a.), with C 1s electron mean free path $(\lambda)=2.9 \mathrm{~nm}$ (value obtained for AlK $\alpha$ X-ray photoemitted electrons ${ }^{47}$ ), $10^{\circ}$ and $90^{\circ}$ should correspond, respectively, to 1.5 and $8.7 \mathrm{~nm}$.
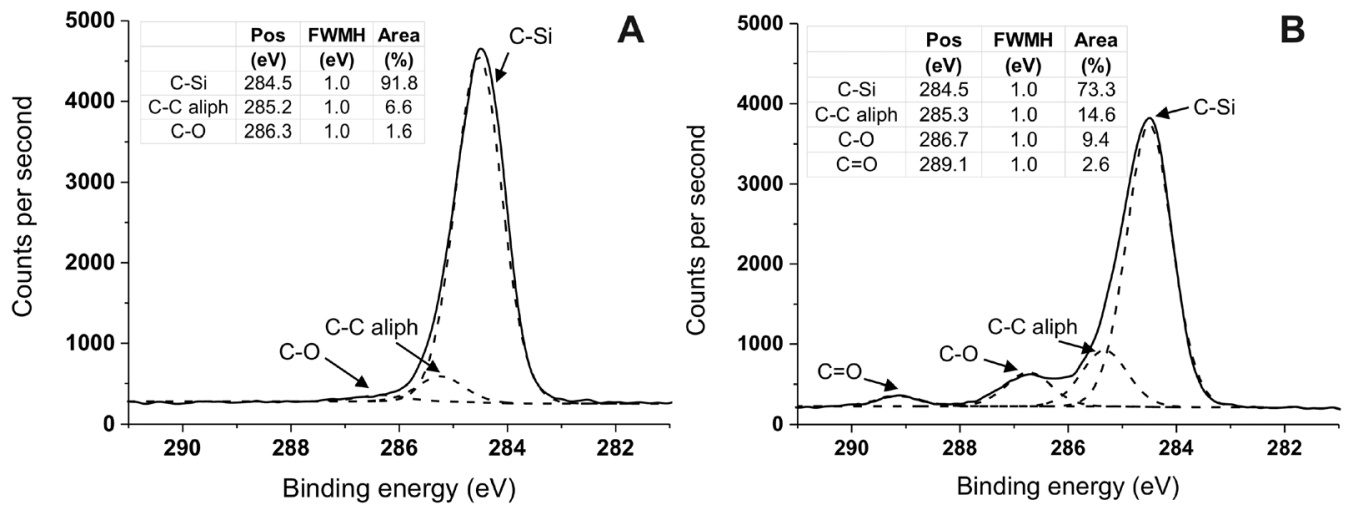

Fig. 4 XPS C 1s high-resolution spectra (t.o.a. $10^{\circ}$ ) of silicone substrate (S10) unmodified (A) and modified with Sil-GMMA1 (B) after storage in water for 14 days. (A) The presence of saturated $C-C$ bonds and of trances of $C-O$ bonds is due to the cross-linkers and silanizing agents used in the Sylgard 184 formulation. (B) The increased amount of $\mathrm{C}-\mathrm{O}$ bonds and the presence of $\mathrm{C}=\mathrm{O}$ groups are ascribed to the ester and alcohol groups of PGMMA chains. Spectra have been charge corrected to position the $\mathrm{C} 1 \mathrm{~s}$ at $284.5 \mathrm{eV}$, which is the binding energy for the binary arrangement $\left(\left(\mathrm{CH}_{3}\right)_{2} \mathrm{SiO}_{2} / 2\right)$ of PDMS. ${ }^{38}$ It is noted that both in (A) and in (B) the binding energy for aliphatic $\mathrm{C}-\mathrm{C}$ is higher than its most usual value (285.0 eV); although a precise explanation for this shift is difficult to find, such a higher binding energy is not unheard of, and has been associated with aliphatic groups present e.g. in peptide-coated silicon, ${ }^{49}$ as well as in diamond-like carbon ${ }^{50}$ or in graphene oxide ${ }^{51}$ or nanotubes $^{52}$ derivatives.

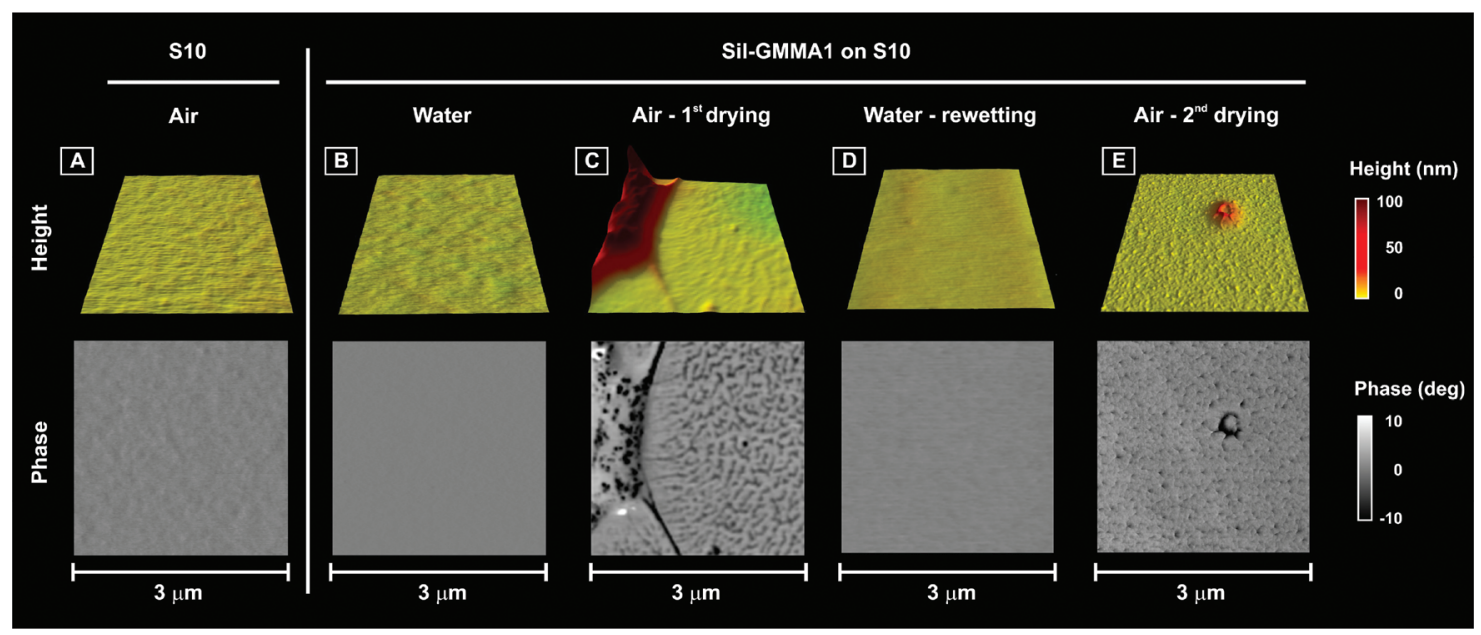

Fig. 5 Height and phase images (tapping mode AFM) of S10 before (A) and after the adsorption of Sil-GMMA1 (B to E). For the latter, images were recorded immediately after adsorption under water after removing the ethanol-water medium (B), in air 24 hours after removal of water (C), again submerged under water for $24 \mathrm{~h}$ after a $26 \mathrm{~h}$ drying cycle in air (D), and finally in air again 24 hours after removal of water (E). Please note that the phase scale uses the median value of the phase as its zero value in each image, and thus scales of different images should be compared only in relative terms. 


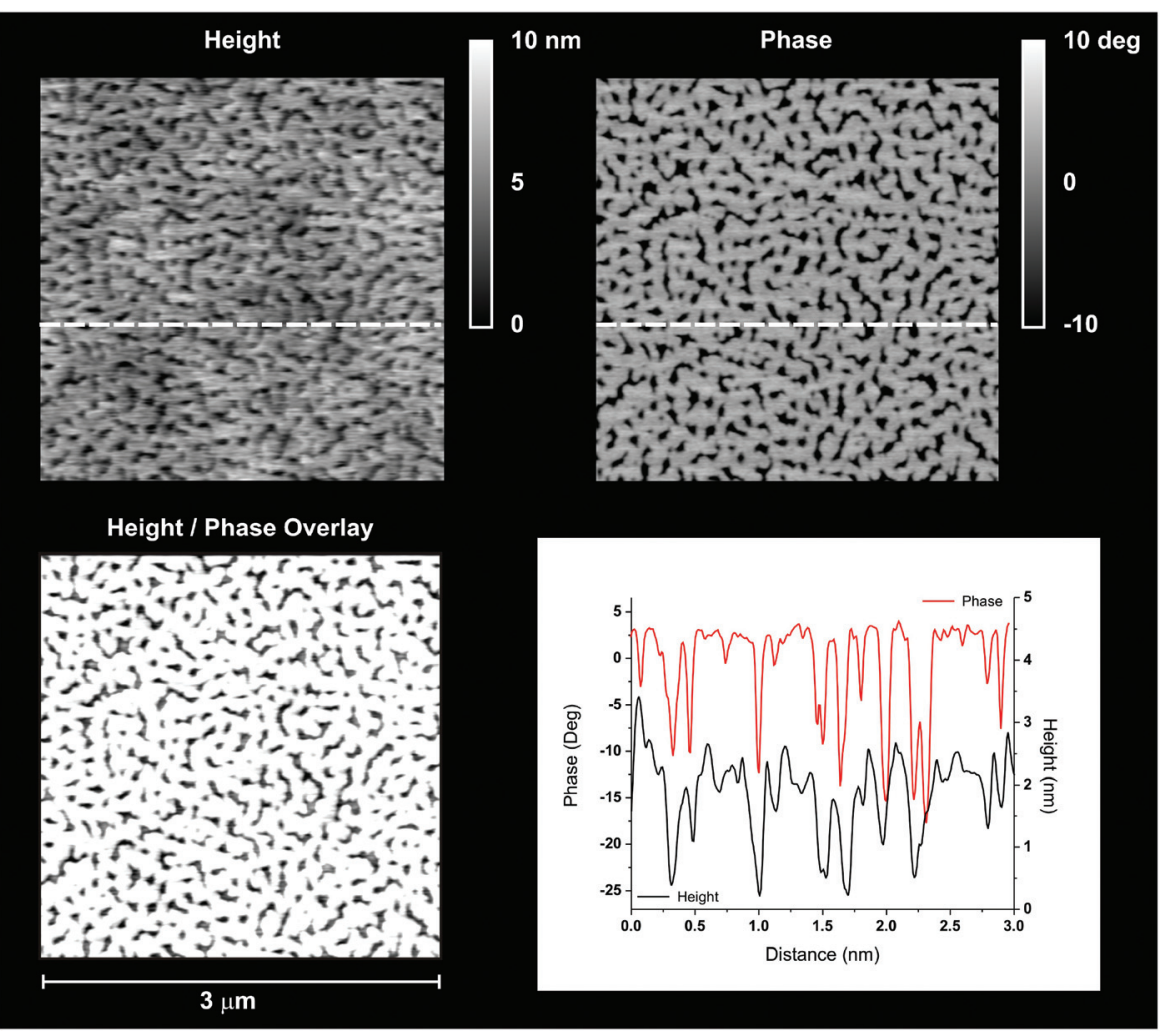

Fig. 6 Comparison of height (top left) and phase (top right) for a $3 \mu \mathrm{m} \times 3 \mu \mathrm{m}$ flat area of Sil-GMMA1-coated S10. The pattern observed is very similar, as can be seen on an overlay of the two pictures (bottom left); a scan of the two images along the dashed white line provides a more quantitative perspective, showing how "higher" and "lower" areas have a height difference of about $2 \mathrm{~nm}$, and the dips in height have a very close correspondence in the phase profile (bottom right).

(A) Morphology. Both flat areas and aggregates maintained their overall roughness and morphology (see ESI, Fig. 5SI; $†$ see also Fig. 7A), but a significant surface reorganization was apparent in the phase images of the latter; in particular, the component characterized by a lower phase (most likely PDMS) appeared to gradually increase its surface presence (Fig. 7B). Since in the same time frame the phase structure of the flat areas did not change appreciably, it seems therefore reasonable to ascribe the wetting/hydrophobic recovery suggested by contact angle measurements to the dynamics of the surface aggregates.

(B) Young's modulus and adhesion force. In air, the average stiffness of the coated surfaces was rather similar to that of the pristine silicone substrate (compare top row Fig. 8A with Fig. 1, middle left) and was substantially unaffected by the drying process. Measurements performed selectively on aggregates showed them to be markedly stiffer than the flat areas (Fig. 8B and $\mathrm{C}$ ), and the latter to have a modulus virtually identical to that of the uncoated silicone. The latter point suggests therefore Sil-GMMA1 to form there a very thin layer in these areas; considering that they cover the vast majority of the surfaces, this confirms the XPS indications. In terms of adhesion of the coated surfaces to the AFM tip (Fig. 8D and E), the sample behavior was again dominated by the flat areas, which are more polar than both the substrates (3-4 times larger adhesion force) and the aggregates. It is noteworthy that the flat areas adhesion showed a small but measurable decrease with time, which may lead to a decrease in contrast in phase images (Fig. 7B); we are inclined to ascribe the two phenomena to the slow evaporation of water from PGMMA blocks.

It seems therefore reasonable to assume the flat areas to be composed of a thin (mono)layer of Sil-GMMA1 that (a) does not markedly affect the mechanical properties of the substrate, but (b) does alter its surface energy by displaying GMMA blocks, and (c) does not undergo a major reorganization during drying, i.e. does not show a hydrophobic recovery.

The aggregates appear to be irregular assemblies of the block copolymers, and they undergo significant restructuring during drying; since they are highly unlikely to derive from the colloidal precursors (they are not recorded under water), they are likely produced during drying. An additional feature would seem to support this hypothesis: most aggregates appeared to be surrounded by $\approx 20 \mathrm{~nm}$-thick layers (Fig. 9A), whose shape seems to recall dewetting/contraction phenomena. During drying, these intermediate regions underwent a surface reorganization similar to that of large aggregates, i.e. with small variations in the profile but very significant time dependency of the phase (Fig. 9B). Unfortunately, the limited lateral resolution of nanoindentation measurements did not allow us to measure the adhesion force selectively in these regions to 


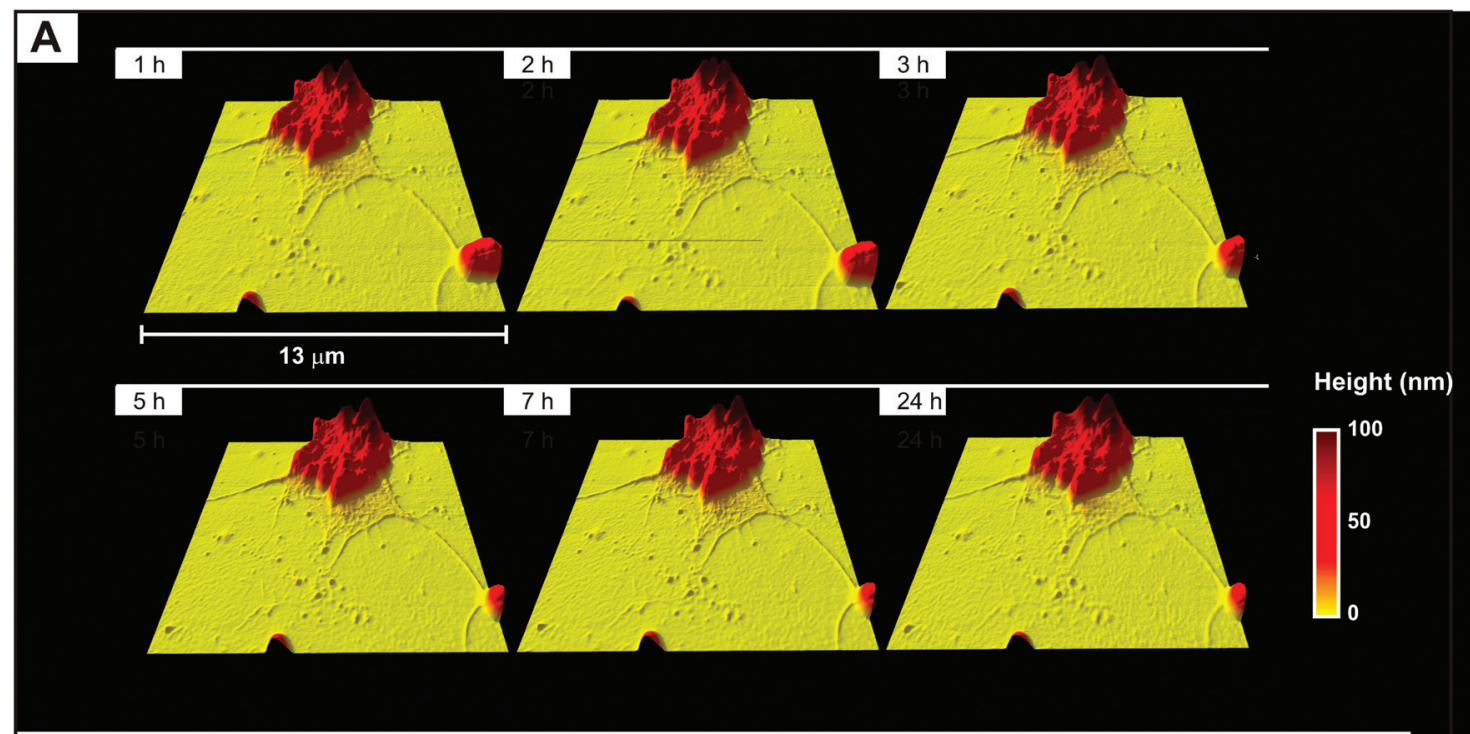

\section{B}
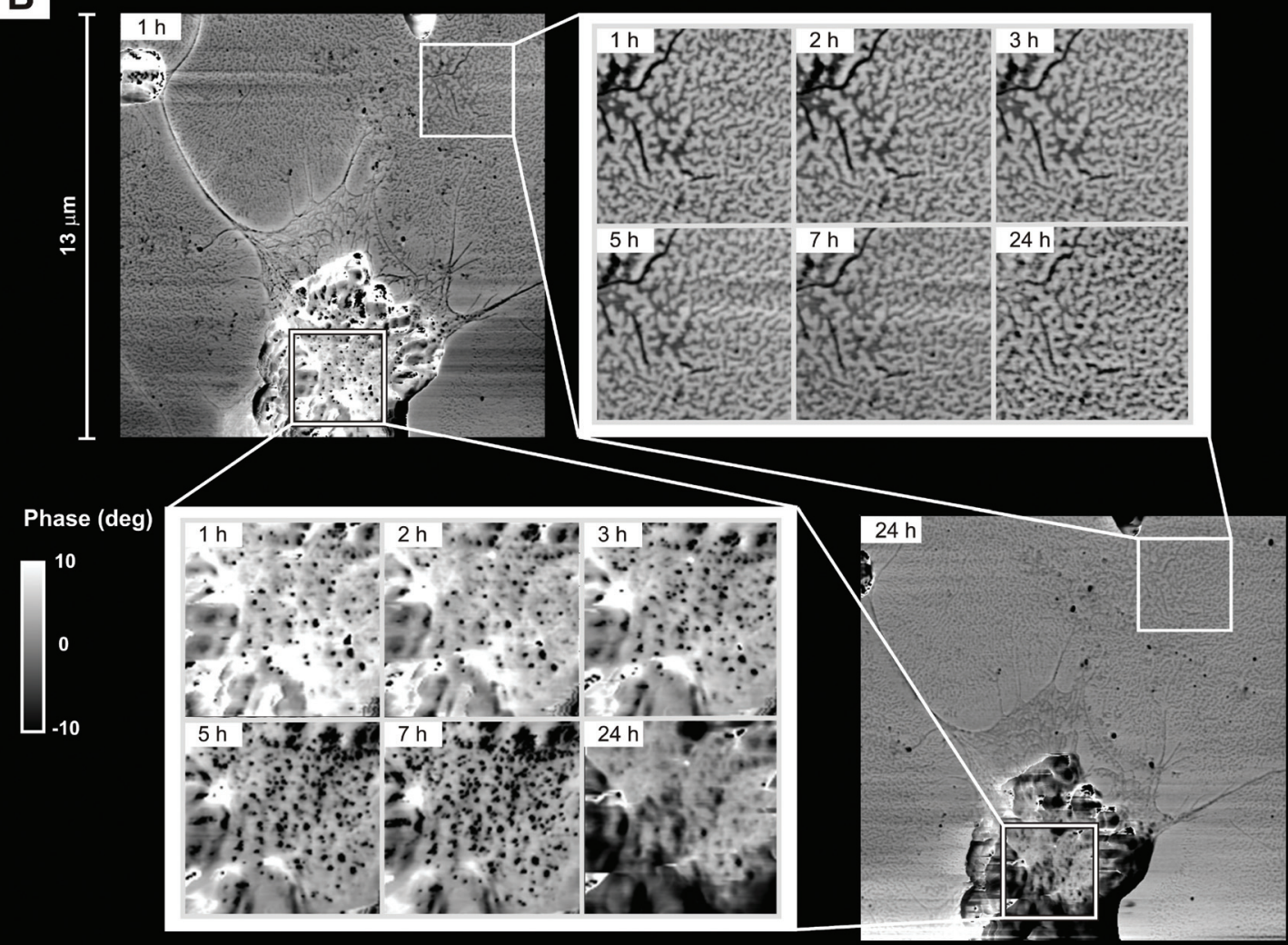

Fig. 7 (A) The morphology of large aggregates did not show any appreciable change during a $24 \mathrm{~h}$ drying cycle. (B) The phase pattern of the flat areas did not undergo major changes during the drying cycle, although it appeared that some short-range reorganization may have occurred (thinning of some "black", likely PDMS domains). On the other hand, the phase images of the large aggregates showed very significant changes, which suggest the migration of lower phase components (likely PDMS blocks) on the surface of the material during drying.

highlight any difference in surface polarity from the aggregates.

We propose here a speculative explanation. If we assume the height of GMMA domains to be around 2-2.5 nm (as suggested by the scans in Fig. 6), we could estimate that the $70 \mathrm{wt} \%$ silicone content would lead to an overall thickness of
7-10 nm for a surface-adsorbed Sil-GMMA1 monolayer; the intermediate regions could be then interpreted as double or oligo-layers, possibly originating from a multilamellar organization of the polymers within the colloids. Indeed, while in water dispersion Sil-GMMA1 forms elongated structures resembling worm-like micelles, in water-ethanol lower-curva- 

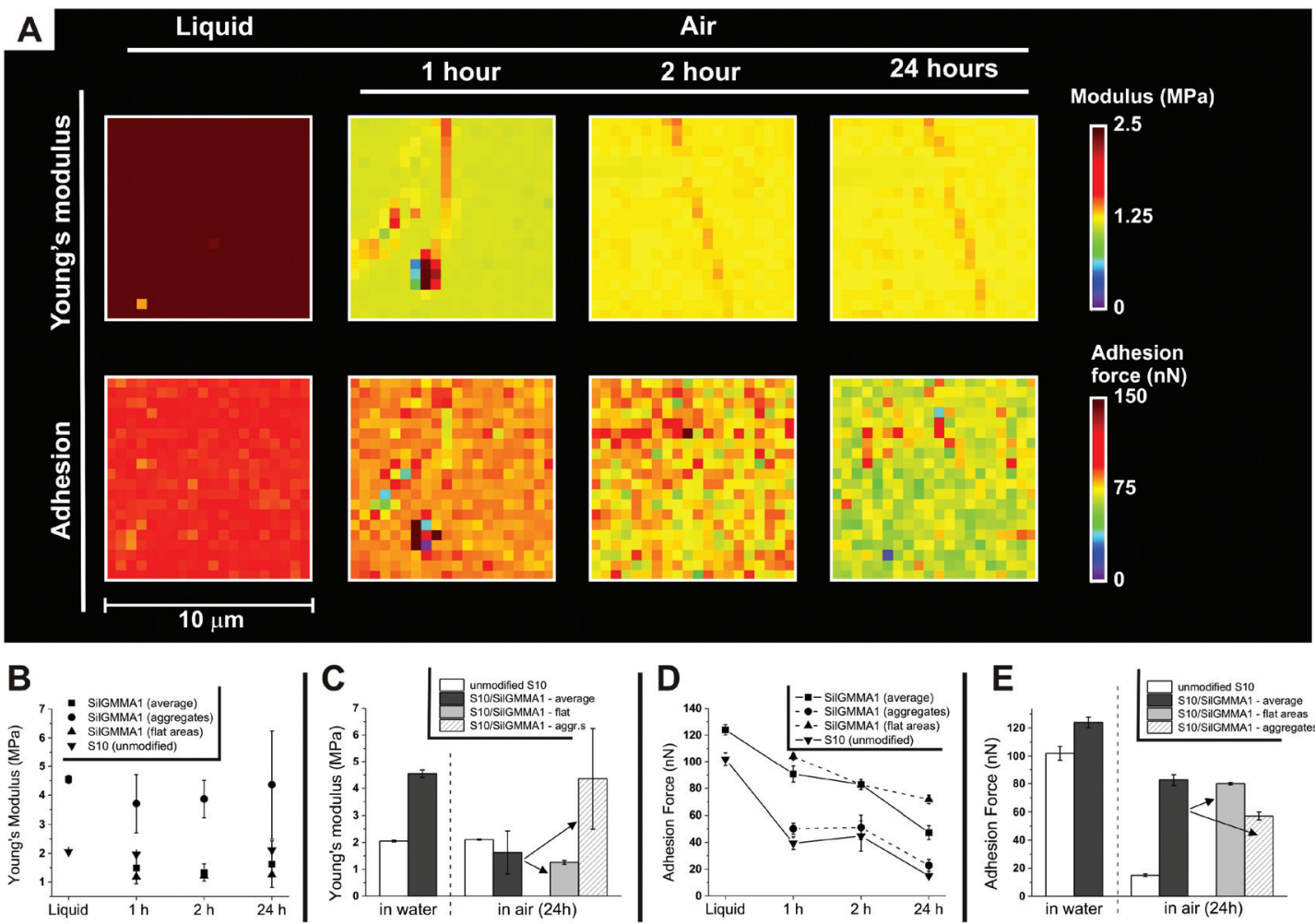

Fig. 8 (A) Typical Young's modulus (above) and adhesion force maps (below) of S10 coated with Sil-GMMA1; the same area was imaged under water and 1, 2 and $24 \mathrm{~h}$ after its removal. For the segmentation process used to distinguish flat areas from aggregates, see ESI, Fig. 7SI. $\dagger$ Please note that the Young's modulus of the coated material under water appears stiffer than in air; this effect was not recorded on the uncoated substrates (Table 1) and likely stems from the choice of the Hertz model to fit nanoindentation data: this model does not consider the substrate surface energy; thus it makes it impossible to quantitatively compare the modulus of a hydrophilic surface in air and under water. (B) Young's modulus averaged over $10 \mu \mathrm{m} \times 10 \mu \mathrm{m}$ areas $(n=3)$ for unmodified and coated S10, in deionized water (liquid) or in air as a function of the drying time. (C) Data from (B), clearer comparison of water/24 $\mathrm{h}$ in air. (D) Adhesion force measured as described in (B). (E) Data from (D), clearer comparison of water/24 $\mathrm{h}$ in air. For a similar comparison of $1 \mathrm{~h}$ and $5 \mathrm{~h}$ drying, see ESI, Fig. $8 \mathrm{SI} . \dagger$

ture e.g. lamellar structures are probably more favoured, due to the reduced solvation of GMMA units.

It could be further hypothesized that while under water all surface is likely covered by multilayer, GMMA-displaying structures, a long-range contraction and re-organisation would occur during drying, eventually yielding aggregates and leaving mostly PDMS-displaying intermediate regions as partial remnants.

\section{Protein adsorption}

The adsorption of (plasma) proteins to the surfaces of synthetic materials is widely recognized as one of the most important causes of cell (platelet, leukocyte) adhesion and ultimately foreign body reaction. Surface heterogeneities, electrostatic or other polar interactions can affect protein adsorption, ${ }^{53,54}$ but on PDMS it is generally accepted that this is mainly driven by hydrophobic effects, i.e. the entropy-driven release of ordered water from silicone surfaces. Therefore, the higher surface polarity of Sil-GMMA-coated surfaces may have a significant influence on the amount and quality of adsorbed proteins.

For this study we have employed bovine serum albumin (BSA) and bovine plasma fibrinogen (BPF), which are used as models for the corresponding human proteins; in humans, albumin is the most abundant plasma protein, with a concentration of about $30-50 \mathrm{mg} \mathrm{mL} \mathrm{m}^{-1}$ (fibrinogen $=1-3 \mathrm{mg} \mathrm{mL}^{-1}$ ) and is one of the first proteins to adsorb on the surface of implanted biomaterials; ${ }^{5-57}$ its deposition is often considered to act as a sort of passivation, since it does not specifically encourage platelet or leukocyte adhesion. In contrast, the adsorption of fibrinogen is well known to trigger undesired reactions. ${ }^{6,7}$

Adsorption experiments were performed on S10 freshly coated with Sil-GMMA1-3; since the samples have not been dried, we assume that at least in the case of Sil-GMMA1 the substrates presented a GMMA-rich surface, possibly in a multilayer morphology (= no aggregates).

The amount of adsorbed protein was first evaluated via the bicinchoninic acid (BCA) assay after desorption with sodium dodecyl sulfate (Fig. 10A and B). Due to the unselective nature of the test, we have separately investigated BSA and BPF, which were used at concentrations similar to those in plasma (resp. 10 and $1 \mathrm{mg} \mathrm{mL}{ }^{-1}$, using $1 \mathrm{mg} \mathrm{mL}^{-1}$ BSA as a control). The presence of Sil-GMMA2 and 3 did not appreciably modify the adsorption of BPF, but increased that of BSA; on the other hand, Sil-GMMA1 affected both, with a $\approx 30 \%$ decrease in BPF 

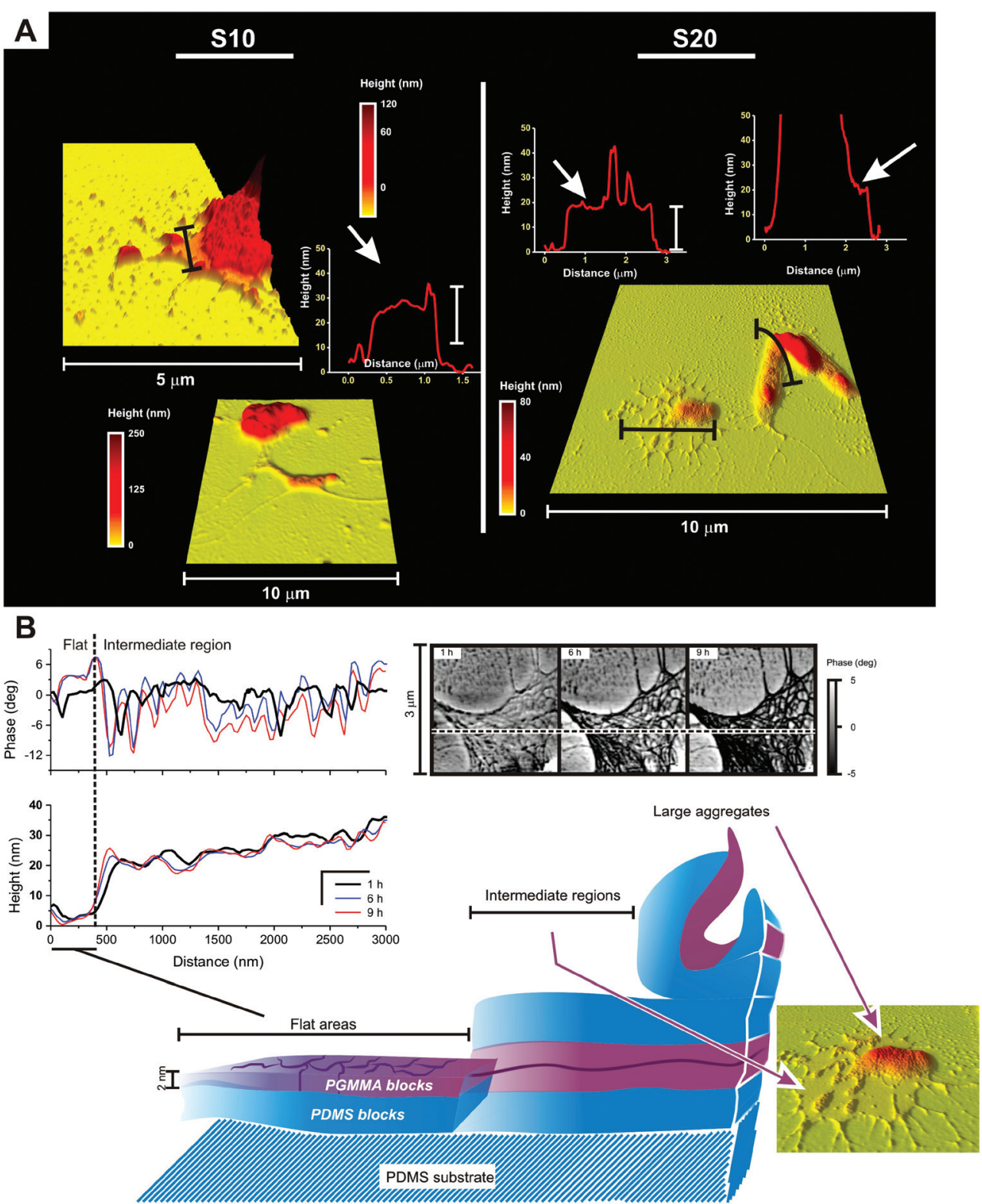

Fig. 9 (A) Details of the morphology of some large aggregates produced by Sil-GMMA1 on S10 and S20 substrates. The black bars in the pictures show the directions of the scans presented in the graphs. For both substrates it is possible to recognize the presence of intermediate, flat regions around the aggregates. These areas appear to have a thickness of around $20 \mathrm{~nm}$ and a roughness of $2-3 \mathrm{~nm}$. (B) Morphology of the intermediate regions on coated silicone substrates (S10). The phase profile of these areas (top left graph and top right pictures; both refer to 1, 6 and $9 \mathrm{~h}$ time points) considerably evolves during the first few hours of drying, with most changes occurring in areas where the height profile records small $(2-3 \mathrm{~nm})$ or negligible modifications; overall, the phase decreases, suggesting the presentation of silicone blocks at the air interface. A proposed structure of the surface elements is provided in the sketch at the bottom of the figure.

and $\mathrm{a} \approx 100 \%$ increase in BSA. This effect is not new: using polyurethane-PGMMA hydrogels Mequanint et al. observed higher albumin and lower fibrinogen adsorption with increasing PGMMA content, ${ }^{27}$ thus showing PGMMA to have preferential interactions with albumin.

We have then used fluorescamine to detect the presence of primary amino groups directly on Sil-GMMA1-coated vs. unmodified S10, without desorption (Fig. 10C). Upon reaction with amines, fluorescamine exhibits maximum emission at $460 \mathrm{~nm}$ when excited at $390 \mathrm{~nm} ;{ }^{58}$ since we have analysed only one type of protein at a time, the fluorescence intensity at $460 \mathrm{~nm}$ is directly proportional to the amount of protein adsorbed; due to the higher sensitivity of the method, it also allowed us to extend the study to more diluted protein solutions. Confirming the results of the BCA assays, at all concentrations we have recorded an increase in BSA and a decrease in BPF adsorption.

Fig. 10D graphically summarizes the comparison of the relative affinity of Sil-GMMA1-coated and unmodified S10 for 

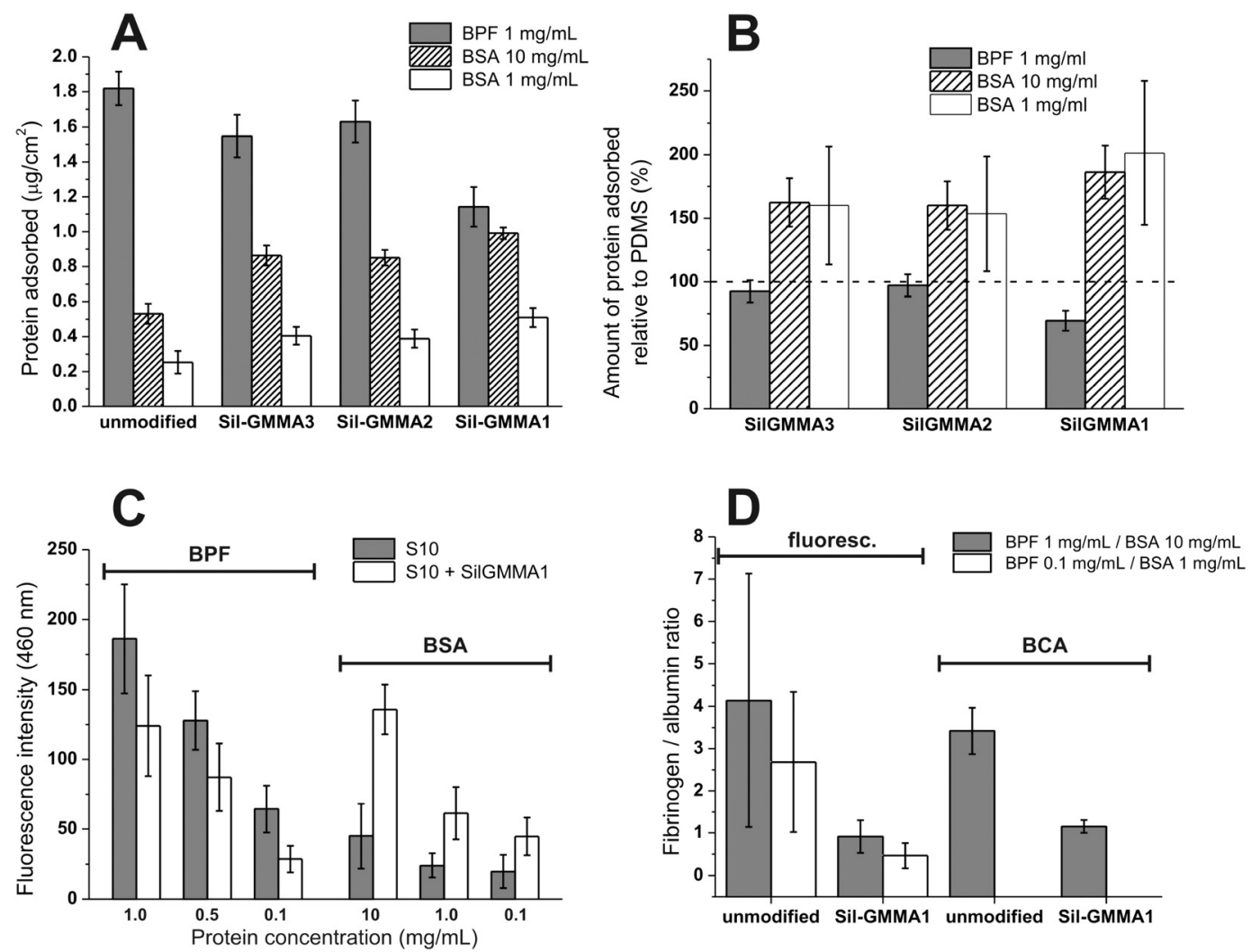

Fig. 10 (A) Amount of protein detached from unmodified and coated S10 and analyzed through the BCA assay; the substrates were incubated for $3 \mathrm{~h}$ in different protein solutions (BPF $1 \mathrm{mg} \mathrm{mL}^{-1}, \mathrm{BSA} 10$ and $1 \mathrm{mg} \mathrm{mL}^{-1}$ ). (B) The data of graph (A) are expressed in terms relative to the unmodified PDMS substrate (S10). (C) Results of the fluorescamine assay (fluorescence intensity at $460 \mathrm{~nm}$ ) on S10 unmodified and modified with Sil-GMMA1 after $3 \mathrm{~h}$ of incubation in different protein solutions (BPF 1, 0.5 and $0.1 \mathrm{mg} \mathrm{mL}^{-1}, \mathrm{BSA} 10,1$ and $0.1 \mathrm{mg} \mathrm{mL}^{-1}$ ). (D) Relative comparison of the affinity of unmodified or Sil-GMMA1-coated S10 for the two proteins. The data are obtained by dividing the BPF fluorescence intensity (fluorescamine assay) or amount per surface area (BCA assay) by the corresponding figures for BSA.

the two proteins, at a fibrinogen/albumin ratio similar to that in blood (1:10); please note that these data refer to individual adsorption experiments and not to competitive binding. Both analytical techniques showed a clear switch from a BPF-philic to a BSA-philic character upon surface coating, which can be promising for decreasing cell attachment and the likelihood of foreign body reaction in vivo.

There are, however, a few caveats. First, adsorbed albumin can be replaced by other proteins having higher affinity for hydrophobic surfaces, like fibrinogen. Obviously, the extent of this effect depends on the nature of the surface. ${ }^{59}$ Therefore studies investigating competitive proteins adsorption or albumin replacement by fibrinogen will be undertaken. Second, protein adsorption may not provide an univocal understanding of the biological performance, since the degree of the biological activity of adsorbed proteins depends on their molecular spreading (degree of unfolding) and/or variations in the orientation or accessibility of the cell binding domains, ${ }^{6,757,60}$ but also on the composition of the adsorbed layer: for example, there is evidence that co-adsorbed albumin influences the "surface biological activity" of fibrinogen. ${ }^{61}$

\section{Conclusions}

We have demonstrated that the physisorption of PDMS-based macroamphiphiles containing hydrophilic PGMMA blocks (SilGMMAs) provides measurable and likely beneficial effects, as long as polymers with high PDMS content are employed. For example, when in a water environment the surface-deposited polymers have a significant effect on protein adsorption, changing the surface character from preferentially fibrinogenadsorbing (in perspective more cell-adhesive) to preferentially albumin-adsorbing (less cell-adhesive). This switch can be very beneficial for the modulation of cell adhesion and activation.

However, the situation becomes complicated in air; the PDMS surface was shown to be predominantly covered by a non-restructuring and thin layer, which showed the characteristics of a phase-segregated monolayer. Although in less conspicuous amounts, other morphologies (aggregates) are also produced in the early stages of drying, and they may derive from the re-organization of multilamellar structures generated in the deposition process. Differently from the monolayers, they undergo significant reorganization (hydrophobic recovery) 
under air and despite their rather low amount we are inclined to ascribe to them a dominant role in the macroscopic contact angle behaviour.

In the perspective of a stable surface functionalization, the presence of the latter structures should be minimized, which likely means to achieve control over the number of layers deposited; this could be possibly achieved by optimizing the concentration of Sil-GMMA1 during the colloidal adsorption, and/ or on the morphology of the colloids themselves, e.g. via sonication or extrusion.

\section{Acknowledgements}

Dr Christopher Cadman and Dr Roberto Donno gratefully acknowledge EPSRC for PhD studentships, the latter in the framework of the EPSRC-funded North West Nanoscience Doctoral Training Centre (NoWNano DTC). Finally, the authors wish to also acknowledge the support from the EPSRC grant EP/F019750/1 (A coordinated open-access centre for comprehensive materials analysis) to perform XPS measurements.

\section{References}

1 K. J. Quinn and J. M. Courtney, Br. Polym. J., 1988, 20, 25-32.

2 X. J. Hao, J. L. Jeffery, J. S. Wilkie, G. F. Meijs, A. B. Clayton, J. D. Watling, A. Ho, V. Fernandez, C. Acosta, H. Yamamoto, M. G. M. Aly, J. M. Parel and T. C. Hughes, Biomaterials, 2010, 31, 8153-8163.

3 E. L. Lawrence and I. G. Turner, Med. Eng. Phys., 2005, 27, 443-453.

4 D. Wolfram, W. Dolores, C. Rainer, R. Christian, H. Niederegger, N. Harald, H. Piza, P. Hildegunde, G. Wick and W. Georg, J. Autoimmun., 2004, 23, 81-91.

5 J. C. McDonald and G. M. Whitesides, Acc. Chem. Res., 2002, 35, 491-499.

6 W. J. Hu, J. W. Eaton and L. P. Tang, Blood, 2001, 98, 12311238.

7 B. Sivaraman and R. A. Latour, Biomaterials, 2010, 31, 832839.

8 A. I. Lopez, A. Kumar, M. R. Planas, Y. Li, T. V. Nguyen and C. Cai, Biomaterials, 2011, 32, 4336-4346.

9 A. L. Hook, C. Y. Chang, J. Yang, S. Atkinson, R. Langer, D. G. Anderson, M. C. Davies, P. Williams and M. R. Alexander, Adv. Mater., 2013, 25, 2542-2547.

10 A. L. Hook, C. Y. Chang, J. Yang, J. Luckett, A. Cockayne, S. Atkinson, Y. Mei, R. Bayston, D. J. Irvine, R. Langer, D. G. Anderson, P. Williams, M. C. Davies and M. R. Alexander, Nat. Biotechnol., 2012, 30, 868-899.

11 H. Chen, Z. Zhang, Y. Chen, M. A. Brook and H. Sheardown, Biomaterials, 2005, 26, 2391-2399.

12 S. Tugulu and H. A. Klok, Macromol. Symp., 2009, 279, 103109.

13 M. H. Wu, Surf. Interface Anal., 2009, 41, 11-16.
14 Z. Y. Zhang, J. C. Wang, Q. Tu, N. Nie, J. Sha, W. M. Liu, R. Liu, Y. R. Zhang and J. Y. Wang, Colloids Surf., B, 2011, 88, 85-92.

15 K. Yu and Y. C. Han, Soft Matter, 2006, 2, 705-709.

16 M. Li, K. G. Neoh, L. Q. Xu, R. Wang, E. T. Kang, T. Lau, D. P. Olszyna and E. Chiong, Langmuir, 2012, 28, 1640816422.

17 T. Rambarran, F. Gonzaga and M. A. Brook, Macromolecules, 2012, 45, 2276-2285.

18 J. J. Wang and F. Liu, J. Mater. Sci. Mater. Med., 2011, 22, 2651-2657.

19 J. H. Seo, R. Matsuno, T. Konno, M. Takai and K. Ishihara, Biomaterials, 2008, 29, 1367-1376.

20 J.-H. Seo, T. Shibayama, M. Takai and K. Ishihara, Soft Matter, 2011, 7, 2968-2976.

21 F. Wan, X. W. Pei, B. Yu, Q. Ye, F. Zhou and Q. J. Xue, ACS Appl. Mater. Interfaces, 2012, 4, 4557-4565.

22 Z. Q. Wu, W. F. Tong, W. W. Jiang, X. L. Liu, Y. W. Wang and H. Chen, Colloids Surf., B, 2012, 96, 37-43.

23 F. Abbasi and H. Mirzadeh, J. Polym. Sci., Part B: Polym. Phys., 2003, 41, 2145-2156.

24 C. Giacomelli, V. Schmidt and R. Borsali, Macromolecules, 2007, 40, 2148-2157.

25 R. Haigh, S. Rimmer and N. J. Fullwood, Biomaterials, 2000, 21, 735-739.

26 C. Johnson, L. Perlin, P. Wyman, B. Zhao, N. J. Fullwood, S. MacNeil and S. Rimmer, in Polymer Networks: Synthesis, Properties, Theory and Applications, Wiley-VCH Verlag Gmbh, Weinheim, 2010, vol. 291-292, pp. 314-325.

27 K. Mequanint, A. Patel and D. Bezuidenhout, Biomacromolecules, 2006, 7, 883-891.

28 E. Patrucco, S. Ouasti, C. D. Vo, P. De Leonardis, A. Pollicino, S. P. Armes, M. Scandola and N. Tirelli, Biomacromolecules, 2009, 10, 3130-3140.

29 S. Rimmer, C. Johnson, B. Zhao, J. Collier, L. Gilmore, S. Sabnis, P. Wyman, C. Sammon, N. J. Fullwood and S. MacNeil, Biomaterials, 2007, 28, 5319-5331.

30 C. Wang, B. Yu, B. Knudsen, J. Harmon, F. Moussy and Y. Moussy, Biomacromolecules, 2008, 9, 561-567.

31 E. P. Everaert, H. C. VanderMei and H. J. Busscher, J. Adhes. Sci. Technol., 1996, 10, 351-359.

32 H. Hillborg and U. W. Gedde, Polymer, 1998, 39, 1991-1998.

33 Z. G. Wu and K. Hjort, Lab Chip, 2009, 9, 1500-1503.

34 J. A. Neff, P. A. Tresco and K. D. Caldwell, Biomaterials, 1999, 20, 2377-2393.

35 C. FreijLarsson, T. Nylander, P. Jannasch and B. Wesslen, Biomaterials, 1996, 17, 2199-2207.

36 S. Lee, R. Iten, M. Muller and N. D. Spencer, Macromolecules, 2004, 37, 8349-8356.

37 G. Robert-Nicoud, R. Evans, C. D. Vo, C. Cadman and N. Tirelli, Polym. Chem., 2013, 4, 3458-3470.

38 L. A. O'Hare, A. Hynes and M. R. Alexander, Surf. Interface Anal., 2007, 39, 926-936.

39 J. L. Hutter and J. Bechhoefer, Rev. Sci. Instrum., 1993, 64, 1868-1873. 
40 J. W. Harding and I. N. Sneddon, Proc. Camb. Philos. Soc., 1945, 41, 16-26.

41 I. N. Sneddon, Int. J. Eng. Sci., 1965, 3, 47-57.

42 C. A. Clifford and M. P. Seah, Appl. Surf. Sci., 2005, 252, 1915-1933.

43 N. Galler, H. Ditlbacher, A. Hohenau, A. Leitner, F. R. Aussenegg and J. R. Krenn, Appl. Phys. B: Lasers Opt., 2011, 104, 931-934.

44 D. J. Campbell, J. A. Olson, C. E. Calderon, P. W. Doolan, E. A. Mengelt, A. B. Ellis and G. C. Lisensky, J. Chem. Educ., 1999, 76, 1205-1211.

45 H. Hillborg, N. Tomczak, A. Olah, H. Schonherr and G. J. Vancso, Langmuir, 2004, 20, 785-794.

46 A. Olah, H. Hillborg and G. J. Vancso, Appl. Surf. Sci., 2005, 239, 410-423.

47 R. F. Roberts, D. L. Allara, C. A. Pryde, D. N. E. Buchanan and N. D. Hobbins, Surf. Interface Anal., 1980, 2, 5-10.

48 G. Beamson and D. Briggs, The XPS of Polymers Database, Surface Spectra Ltd, 2000.

49 S. S. Sam, J.-N. J. N. Chazalviel, A. C. A. C. GougetLaemmel, F. F. Ozanam, A. A. Etcheberry and N.-e. N. Gabouze, Nanoscale Res. Lett., 2011, 6, 412.

50 P. Merel, M. Tabbal, M. Chaker, S. Moisa and J. Margot, Appl. Surf. Sci., 1998, 136, 105-110.
51 R. Rozada, J. I. Paredes, S. Villar-Rodil, A. Martinez-Alonso and J. M. D. Tascon, Nano Res., 2013, 6, 216-233.

52 H.-L. Hsu, I. J. Teng, Y.-C. Chen, W.-L. Hsu, Y.-T. Lee, S.-J. Yen, H.-C. Su, S.-R. Yeh, H. Chen and T.-R. Yew, Adv. Mater., 2010, 22, 2177-2181.

53 J. D. Andrade and V. Hlady, Adv. Polym. Sci., 1986, 79, 1-63. 54 D. L. Elbert and J. A. Hubbell, Annu. Rev. Mater. Sci., 1996, 26, 365-394.

55 I. C. Goncalves, M. C. L. Martins, J. N. Barbosa, P. Oliveira, M. A. Barbosa and B. D. Ratner, J. Mater. Sci. Mater. Med., 2011, 22, 2053-2063.

56 K. Kottkemarchant, J. M. Anderson, Y. Umemura and R. E. Marchant, Biomaterials, 1989, 10, 147-155.

57 B. Sivaraman and R. A. Latour, Biomaterials, 2010, 31, 1036-1044.

58 S. Udenfrie, S. Stein, P. Bohlen and W. Dairman, Science, 1972, 178, 871-872.

59 S. J. Geelhood, T. A. Horbett, W. K. Ward, M. D. Wood and M. J. Quinn, J. Biomed. Mater. Res., Part B, 2007, 81B, 251260.

60 M. E. Soderquist and A. G. Walton, J. Colloid Interface Sci., 1980, 75, 386-397.

61 J. A. Chinn, S. E. Posso, T. A. Horbett and B. D. Ratner, J. Biomed. Mater. Res., 1992, 26, 757-778. 